\title{
Übersichtsarbeit
}

\section{Nichtärztliche Assistenzberufe mit originär ärztlichen Tätigkeiten}

\author{
Albrecht Wienke $^{1^{\star}}$, K. Janke ${ }^{1}$ \\ ${ }^{1}$ Wienke \&Becker \\ GMS Mitteilungen aus der AWMF 2006;3:Doc31
}

\section{Text}

\section{Einleitung}

Anlässlich der jüngsten Ärztetage hat der Vorstand der Bundesärztekammer in seinen Tätigkeitsberichten regelmäßig auf die Entwicklung der Arztzahlen in Klinik und Praxis hingewiesen (www.bundesaerztekammer.de). Dabei trat zunehmend die Erkenntnis zu Tage, dass die Ergebnisse der Ärztestatistik eine erhebliche Unterversorgung gerade im Bereich der niedergelassenen Ärzte belegen, die sich insbesondere in der hausärztlichen Versorgung in ländlich geprägten Gebieten und in den östlichen Bundesländern zeigen. Es verwundert daher nicht, dass als Folge dieser Entwicklung allerorten Gegenmaßnahmen diskutiert werden, die auch von der Bundesärztekammer zum Teil ablehnend kritisch (Family Health Nurse - FHN), zum Teil aber auch positiv (Zusatzqualifizierung der Arzthelferin) kommentiert werden. Zwischen diesen Positionen haben sich mittlerweile erstaunlicherweise recht unbemerkt bereits eine ganze Reihe von nichtärztlichen Assistenzberufen entwickelt, die dabei sind, sich mit oder ohne staatliche Anerkennung weiter zu etablieren. Die aktuellen Diskussionen (vgl. Deutsches Ärzteblatt, Jg. 103, Heft 26, 30. Juni 2006, A 1802 ff.) um nichtärztliche Chirurgieassistenten (Operations-Technischer-Assistent - OTA, Chirurgisch-TechnischerAssistent - CTA) werfen schnell Fragen der Einsatzmöglichkeiten und der rechtlichen Voraussetzungen auf, die im Folgenden näher beleuchtet werden sollen:

\section{Operations-Technische Assistenten (OTA)}

Seit 1990 hat sich eine staatlich bisher nicht anerkannte Ausbildung zum OTA entwickelt. Die dreijährige Ausbildung findet außerhalb des dualen Berufsbildungssystems (System der Berufsschulen) statt, in der Regel an einer der 70 OTA-Schulen, denen sich ca. 450 Krankenhäuser mit 1.800 Ausbildungsplätzen angeschlossen haben. Im Jahr 1996 hat die Deutsche Krankenhausgesellschaft eine „Empfehlung zur Ausbildung und Prüfung von Operationstechnischen Assistentinnen/Assistenten" veröffentlicht (vgl. www.ota.de). Solange keine bundesweite bzw. landesweite Regelung der Ausbildung besteht, soll der Ablauf der Ausbildung dadurch bundesweit vereinheitlicht und standardisiert werden. Zulassungsvoraussetzung für die Schüler ist unter anderem die einjährige Qualifikation zum Krankenpflegehelfer; bei vorheriger Ausbildung zum Arzthelfer oder Krankenpfleger reduziert sich die OTA-Ausbildungszeit. Der Absolvent einer Ausbildung an einer von der DKG anerkannten Schule ist anschließend berechtigt, die Bezeichnung „Operationstechnische Assistentin/Assistent (DKG)“ zu führen. Sein Aufgabengebiet umfasst u.a. die fachkundige Vorbereitung von

"Corresponding Author:

Dr. Albrecht Wienke, Bonner Str. 323, 50968 Köln, eMail: AWienke@Kanzlei-WBK.de 
Instrumenten und Geräten vor der Operation, die Assistenz während der Operation sowie die Nachbereitung der Operation (Entsorgung und Sterilisation der Instrumente); Einzelheiten sind in $\S 1$ Abs. 2 der DKG-Empfehlung geregelt.

In Schleswig-Holstein wurde 2004 die Landesverordnungen über die Berufsausbildung zur oder zum Operationstechnischen Angestellten erlassen, mit der eine Ausbildung zum OTA in einem dualen System mit staatlicher Anerkennung über das Berufsbildungsgesetz eingeführt wurde. Zugleich wurde die DKG-Empfehlung gegenstandslos. Die Ausgestaltung und die Anforderungen weichen allerdings in wesentlichen Punkten von der Empfehlung der DKG ab, insbesondere in Hinblick auf die ausbildende Person (DKG: Krankenhaus; VO Schleswig-Holstein: Arzt) und die theoretischen Unterrichtsstunden (DKG: 1600-1700; VO Schleswig-Holstein: 840). Wegen des abweichenden Qualifikationsprofils und den geringen Zugangsvoraussetzungen stößt die landesrechtliche Regelung sowohl bei den Kliniken in Schleswig-Holstein als auch bei der DKG auf Kritik.

Die Bundesärztekammer (BÄK) hat in ihrem Tätigkeitsbericht 2004 zugestanden, dass die DKG-Ausbildung sehr erfolgreich war, sah aber keinen Handlungsbedarf. Auch im Tätigkeitsbericht 2005 übt die BÄK Zurückhaltung gegenüber neuen Assistenzberufen und beschränkt sich auf die Frage der delegierbaren Leistungen.

Auf Seiten der Chirurgen ist dagegen eine positive Grundstimmung hinsichtlich der OTAssistenten festzustellen. So hat Prof. Ulrich in den Mitteilungen 2/05 der Deutschen Gesellschaft für Chirurgie die Akzeptanz des Berufsbildes der OTA von Krankenhäusern und von Chirurgen bestätigt. Die Nachfrage der Krankenhäuser nach OTA steige zunehmend und in vielen Kliniken würden bestimmte Operationsschritte unter Aufsicht den Operationsschwestern oder OTAs überantwortet. Auch Prof. Schweiger berichtet in den Mitteilungen 04/05 der Deutschen Gesellschaft für Chirurgie von den durchweg positiven Erfahrungen beim Einsatz von medizinischen Assistenten.

Die Gewerkschaft für Beschäftigte im Gesundheitsdienst kritisiert die Schaffung des Berufes des OTA in hohem Maße und sieht darin die Vernichtung von Arbeitsplätzen für Pflegepersonal, eine „Billiglohngruppe“ und einen „Beruf ohne Zukunft“.

Die Gesundheitsministerkonferenz der Länder sieht dagegen Handlungsbedarf für eine einheitliche Ausbildungsregelung auf Grundlage des Art. 74 Nr. 19 GG und hat mit Beschluss vom 30.6.2006 die Bundesgesundheitsministerin gebeten, einen Referentenentwurf zur bundesrechtlichen Regelung des Berufsbildes des OTA zu erarbeiten und die Finanzierung nach dem KHG sicherzustellen.

\section{Chirurgisch-Technische Assistenten (CTA)}

Seit Anfang 2006 wird die Ausbildung zum Chirurgisch-Technischen Assistenten als Pilotprojekt von der Akademie für operative Aus- und Weiterbildungsberufe der Kaiserswerther Diakonie in Düsseldorf angeboten. Die Ausbildung dauert 3 Jahre und als Zulassungsvoraussetzung wird die Fachhochschulreife in Verbindung mit einem erlernten Beruf (Krankenpfleger, OTA) oder das Abitur verlangt. Die Lehrinhalte richten sich nach einem Tätigkeitskatalog der Deutschen Gesellschaft für Chirurgie. Unter Aufsicht und Verantwortung des Chirurgen sollen die CT-Assistenten u.a. Aufgaben der Patientenlagerung, des OP-Zugangs und des Wundverschlusses übernehmen.

Die Ausbildung wurde u. a. durch die Initiative der Deutschen Gesellschaft für Chirurgie und insbesondere Herrn Prof. Ulrich (ehemaliger Präsident der DGCH), der im Jahr 2004 bereits eine Anfrage hinsichtlich der CTA-Ausbildung an das BMGS gestellt hatte, ins Leben gerufen. Das BMGS hatte seinerzeit Zweifel angemeldet, ob neben den OTAs für eine weitere Fachkraft im Operationsdienst Bedarf bestünde. Eine Abklärung über die Gesetzgebungsbedarf des Bundes solle mit den Ländern erfolgen. Die Bundsärztekammer schlug Bedarfsanalysen vor. 
Die Bildungsstätte des Marienhospitals Osnabrück bietet eine Fortbildung zum „ChirurgieAssistent in der Allgemeinen Chirurgie, Unfallchirurgie und Gynäkologie“ für Krankenschwestern, Fachkrankenschwestern aber auch OTAs mit 2jähriger Berufserfahrung im Operationsdienst an.

\section{Anästhesie-Technische Assistenten (ATA)}

In einem Pilotprojekt bietet das Ausbildungszentrum für Gesundheitsfachberufe des Universitätsklinikums Halle-Wittenberg/Saale eine Ausbildung zum „Anästhesie-technischen Assistent (ATA)“ an. Zulassungsvoraussetzung ist u.a. die Qualifikation als Krankenpflegehelfer, aber keine weitere pflegerische Ausbildung. Die HELIOS-Kliniken GmbH ermöglicht die Qualifikation zum „Medizinischen Assistent für Anästhesie (MAFA)“, allerdings handelt es sich dabei um eine Weiterbildung von Krankenpflegepersonal.

In einer gemeinsamen Stellungnahme des Berufsverbandes Deutscher Anästhesisten und der Deutschen Gesellschaft für Anästhesie und Intensivmedizin wird die starke Stellung des Assistenzpersonals und deren Notwendigkeit zur Vorbereitung, Durchführung und Nachbereitung von Narkosen betont. Allerdings wird absichtlich keine klare Aussage darüber getroffen, ob die Implementierung des ATA ökonomisch vorteilhaft und berufspolitisch sinnvoll, noch ob die Weiterqualifizierung zu MAFA notwendig ist.

\section{Gefäßassistent/-in DGG}

Die Deutsche Gesellschaft für Gefäßchirurgie hat aufgrund der positiven Erfahrungen mit dem Einsatz medizinischer Assistenten Anfang 2006 in Eigenregie ein Weiterbildungskonzept zum Gefäßassistenten für Angehörige der medizinischen Assistenzberufe entwickelt. Der Gefäßassistent soll demnach auf der Basis fundierter Kenntnisse in der Gefäßmedizin nicht zwingend ärztliche Aufgaben erbringen. Er ist sowohl fachlich als auch organisatorisch dem Gefäßchirurgen unterstellt und handelt auf ärztliche Weisung.

\section{Family Health Nurse (FHN)}

Nach dem politisch diskutierten Konzept für den Nurse-Practitioner soll eine Family Health Nurse (FHN) losgelöst von dem Verantwortungsbereich der niedergelassenen Ärzte, freiberuflich und in Konkurrenz zu oder neben Ärzten in der ambulanten Versorgung tätig werden. Im Tätigkeitsbericht der Bundesärztekammer 2005 wird dargelegt, dass sich die Deutsche Akademie für Allgemeinmedizin mit den Einsatzmöglichkeiten der FHN im deutschen Gesundheitswesen befasst. Eine vom BMGS beauftragte Expertengruppe sehe den Einsatz der FHN im Rahmen der Pflege, des Entlassungsmanagements nach stationärer Versorgung und als Pflegeexpertin der Hausarztpraxis als verfolgenswert an. Die Akademie lehnt das Gesamtprojekt der FHN allerdings ab und sieht das Konzept der Zusatzqualifikation der Arzthelferin für die haus- und vertragsärztliche Versorgung als sachgerechter an. Die Hausärzte sollen von delegationsfähigen Leistungen befreit werden, indem bestimmte Schulungs-, pflegerische oder sonstige Leistungen auf die Arzthelferin übertragen werden, die allerdings im Verantwortungsbereich des Arztes verbleibt. Dazu soll eine geeignete Bezeichnung wie z. B. „Hausarztassistentin“ gefunden werden.

\section{Delegationsfähigkeit ärztlicher Leistungen auf nichtärztliches Personal}

Für alle dargestellten und sich ggf. noch entwickelnden Berufsgruppen stellt sich die Frage der Einsatzmöglichkeiten im Rahmen der medizinischen Versorgung, insbesondere danach, ob und welche ärztlichen Leistungen unter welchen Voraussetzungen an solche Berufsgruppen delegiert werden dürfen und ob der Arztvorbehalt durch die Implementierung solcher Arzthilfsberufe im Gesundheitswesen aufgeweicht wird. 
Generell ist unter rechtlichen Aspekten zur Delegierbarkeit ärztlicher Leistungen Folgendes auszuführen:

Grundsätzlich nicht delegationsfähig sind nach Dienstvertragsrecht und unter Berücksichtigung der gemeinsamen Auffassungen der Krankenkassen und der Kassenärztlichen Bundesvereinigung sowie der Bundesärztekammer über die Anforderungen an die persönliche Leistungserbringung Verrichtungen, die wegen ihrer Schwierigkeiten, ihrer Gefährlichkeit oder wegen der Unvorhersehbarkeit etwaiger Reaktionen ärztliches Fachwissen voraussetzen und deshalb vom Arzt persönlich, also eigenhändig durchzuführen sind. Hierzu zählen insbesondere alle operativen Eingriffe, schwierige Injektionen, Infusionen und Blutentnahmen sowie ärztliche Untersuchungen, Diagnostik und die ärztliche Beratung des Patienten.

Demgegenüber besteht Einigkeit, dass es generell delegationsfähige ärztliche Leistungen gibt. Zu diesen Leistungen gehören insbesondere Laborleistungen, Dauerkatheterwechsel und der Wechsel einfacher Verbände sowie radiologische Leistungen.

Schließlich wird differenziert in eine Gruppe der im Einzelfall delegationsfähigen Leistungen. Es gibt regelmäßig Leistungen, die nur im Einzelfall zur Ausführung an besondere nicht ärztliche Mitarbeiter übertragen werden dürfen. Hierzu gehören insbesondere die Injektionen, Infusionen und Blutentnahmen. Der Arzt darf im Einzelfall qualifizierte nicht ärztliche Mitarbeiter mit solchen Tätigkeiten betrauen, sofern sein persönliches Tätigwerden nach Art und Schwere des Krankheitsbildes oder des Eingriffs nicht erforderlich ist und der Mitarbeiter die erforderliche Qualifikation, Zuverlässigkeit und Erfahrung aufweist.

Soweit diese grundlegenden Abgrenzungen in der juristischen Literatur und in der Rechtsprechung unstrittig sind, so streitig sind doch die hierzu bisher beurteilten Einzelfälle:

Der Bundesgerichtshof $(\mathrm{BGH})$ hat bisher noch nicht ausdrücklich und umfassend darüber entscheiden, ob und welche Injektionen der Arzt an nicht ärztliche Mitarbeiter delegieren darf. Nach einem Urteil aus dem Jahre 1959 darf eine examinierte, also voll ausgebildete und geprüfte Krankenschwester intramuskuläre Injektionen nur übertragen erhalten, wenn der Leitende Arzt sich vergewissert, dass die Krankenschwester ihren Aufgaben gewachsen und daneben für ihre Kontrolle durch die vorhandenen Ärzte Sorge getragen ist. In einem Urteil aus dem Jahre 1979 neigte der BGH zu der Ansicht, intramuskuläre Injektionen durch Krankenpflegerhelferinnen ließen sich grundsätzlich nicht akzeptieren, weil Applikationsfehler zu typischen schwerwiegenden Schäden, wie Lähmungen und Spritzenabszessen, führen könnten. Allerdings gibt es, wie die Praxis zeigt, auch unter den Arzt- und Krankenpflegerhelferinnen und Altenpflegern langjährig tätige, verantwortungsbewusste und sorgfältig arbeitende Personen, die unter ärztlicher Anleitung diejenigen Kenntnisse und Fertigkeiten erworben haben, die es dem Arzt erlauben, in geeigneten Einzelfällen Blutentnahmen, intravenöse Injektionen und Infusionen an nicht ärztliches Personal zu delegieren.

Immer setzt die Delegation einer ärztlich angeordneten Injektion oder Infusion die fachliche Qualifikation, also das ausreichende Wissen und hinlängliche Erfahrung des nicht-ärztlichen Mitarbeiters voraus, wovon sich der Arzt zuvor persönlich regelmäßig durch Stichproben überzeugen muss.

Glaubt die jeweils vom Arzt zur Ausübung der delegationsfähigen Leistung angewiesene nicht ärztliche Mitarbeiterin selbst allerdings, dass sie der an sie gestellten Aufgabe nicht gewachsen ist, weil sie gegebenenfalls zu hohe Risiken birgt, wird durch diese Einstellung in den meisten Fällen die notwendige Überprüfung des Qualifikationsgrades der nicht ärztlicher Mitarbeiterin in Frage gestellt. Der Arzt weiß dann, dass es ein gewisses Risiko wäre, die grundsätzlich delegationsfähige Leistung im Einzelfall an eine Mitarbeiterin zu übertragen, die sich selbst nicht für entsprechend qualifiziert oder „,bereit“ hält. Eine Delegation muss also im Einzelfall dann unterbleiben, wenn die Pflegekraft oder der nicht-ärztliche Assistent sich aus eigener Einschätzung selbst nicht in der Lage sieht, die grundsätzlich delegierbare Leistung auszuführen. Eine Delegation muss dann unterbleiben.

Diese in der Rechtsprechung bisher gewonnenen und zugegebenermaßen im Einzelfall nach wie vor strittigen Abgrenzungskriterien lassen sich auf die eingangs dargestellten Assistenz- 
berufe übertragen. Danach ist jedenfalls nach dem derzeitigen Stand der höchstrichterlichen Rechtsprechung mit einer sehr restriktiven rechtlichen Beurteilung zu rechnen. Dies bedeutet, dass die rechtlichen Rahmenbedingungen es derzeit nicht erlauben, den Arztvorbehalt im Rahmen der medizinischen Diagnostik und Therapie durch einen sachlich ausgeweiteten Einsatz nicht-ärztlichen Assistenzpersonals einzuschränken. Es ist vielmehr damit zu rechnen, dass die Rechtsprechung die bisher bereits herangezogenen strengen Maßstäbe auch bei der Etablierung und dem Einsatz neuer Assistenzberufe anlegen wird, so dass die Delegation ärztlicher Leistungen auf nicht-ärztliches Personal auch nur eingeschränkt dem beschriebenen Problem der ärztlichen Unterversorgung wird begegnen können.

Köln, im Oktober 2006 